Aus dem histologischen Laboratorium der Universität Kasan (Prof. C. Arnstein).

\title{
Zur Innervation der Harnblase ${ }^{1}$ ).
}

\author{
Von
}

Dr. N. Grünstein.

Hierzu Tafel I.

Die Lehre vou der Innervation der verschiedenen Organe hat im letzten Decennium ganz bedeutende Fortschritte gemacht dank der Entdeckung der Ehrlich'schen Methylenblauinjection und den Verbesserungen, welche an dieser Methode von C. Arnstein (2) und seinen Schïlern Smirnow und A. Dogiel vorgenommen worden sind. Seitdem es diesen Forschern gelungen ist, Mittel zu entdecken, welche es gestatteten, die sonst leicht verschwindende blaue Färbung der nervösen Elemente auf die Dauer zu fixiren (Pikrocarmin und pikrinsaures Ammoniak), ist die bistologische Technik um eine für das Stadium der Nervenapparate und Nervenendigungen in den perjpheren Organen unersetzliche Methode bereichert worden.

Wir besitzen auch bereits eine ganze Anzahl von Untersuchungen, die vorzüglich mit dieser Methode ansgefülrt, die Frage über die Innervation mehrerer Organe bedeutend gefördert haben. Wir weisen hier nur auf die aus dem hiesigen Laboratorium stammenden Arbeiten hin, auf die Untersuchungen von A. Dogiel, Smirnow (3), A gababow (4), Timof ejew $(5$ a. 6) and Plosebko (7), die uns den Werth der in Rede stehenden Methode zur Genüge erkennen lassen.

1) Die vorliegende Untersuchung ist bereits vor längerer Zeit ausgeführt. In der durch äussere Verhältnisse nicht zu Stande gekommenen Absicht dieselbe fortzusetzen, wurde die Publication bis auf den heutigen Tag verschoben.

Arch. f. mikrosk. Anat. Bd. 55 
Im Folgenden sollen kurz die Resultate meiner Untersuchungen aber die Innervation der Harnblase mitgetheilt werden, die ich auf Anrathen und unter Leitung des hochverebrten Herrn Prof. C. Arnste in im hiesigen Laboratorium angestellt babe.

Zur Färbung der nervösen Elemente wurde fast ausschliesslich die $\mathrm{Ehr}$ li ch'sche Methode angewandt, d. b. es wurde dem durch Chloroform tief betäubten, aber noch athmenden Thiere eine erwärmte $1 \%$ ige Lösung von Methylenblau (Grüble er) in physiologischer Kocbsalzlösung injicirt. Die Injection geschah beim Frosch durch die V. abdominalis, bci kleinen Säugern durch das Herz, bei grössern durch die Aorta. Nach 20 Minuten, resp. einer Stunde wurde die Bauchhöhle eröffnet, die Harnblase herausgeschnitten und unter dem Mikroskop das Eintreten der maximalen Färbung abgewartet, was gewöhnlich nach einer halben oder ganzen Stunde einzutreten pflegte. Darauf kam die Blase auf 24 Stunden in eine kalt gesättigte wässerige Lösung von pikrinsaurem Ammoniak und von da aus zur Authellung in eine Lösung von folgender Zusammensetzung:

Ammonium pikronitric. (obige Lösung) $8 \mathrm{Th}$.

Glycerin

$12 n$

Aq. destill.

$12 \pi$

In dieser Mischung können die Präparate beliebig lange aufbewahrt werden.

\section{Der Nervenapparat der Froschharnblase.}

Aus der makroskopischen Anatomie wissen wir, dass die Harnblase des Frosches sowohl vom cerebrospinalen als auch vom sympathischen Nervensystem innervirt wird. Beiderlei Fasern bilden ein dünnes Nervenstämmchen, das unmittelbar unter der Vereinigungsstelle des achten und neunten Spinalnerven vom Plexus ischio-s. sacrococcygeus abgeht. Ansserdem giebt es noch in der Harnblasenwand eine grosse Anzahl von automatischen Nervenapparaten, die sich in Gestalt von grösseren oder kleineren Nervenknoten präsentiren. Ein solches Ganglion besteht, unter dem Mikroskop betrachtet, aus Nervenzellen und Nervenfasern. Die Zellen sind von runder oder ovaler Form und besitzen einen oder zwei Ausläufer. Sowohl diese als auch die Zellkörper lassen sich nur schwer, ev. gar nicht mit Methylenblau färben, man erkennt sie alsdann nur an dem in ibrem Innern eingeschlossenen 
gelben resp. braunen Pigmente. Dagegen färbt sich jedesmal wunderschön das die Zelle umspinnende Flechtwerk von feinen varikösen Fäden - der pericelluläre Apparat. Um diesen genauer zu betrachten, suche man sich eine Zelle heraus, die nicht in einem Ganglion, sondern isolirt in der Blasenwand liegt. Solche Zellen giebt es in der Harnblase des Frosches in grosser Menge. In Fig. 1 ist eine solche isolirt liegende Zelle abgebildet. Sie ist ziemlich gross unipolar und besitzt einen die ganze Oberfläche der Zelle einnehmenden, prächtig gefärbten pericellulären Nervenapparat und geraden Fortsatz. Letzterer lässt sich scharf von der Faser unterscheiden, die pericellulär endigt, einmal durch die entgegengesetzte Richtung der beiden Fasern, dann durch ihre Fürbung (die pericellulär endigende Faser ist blau violett gefürbt, der gerade Fortsatz roth violett). Endlich ist noch ein Unterschied in der Dicke der beiden Fasern vorhanden: die erstere ist dünn und unterscheidet sich durch nichts von den anderen blassen Fasern des Nervenstämmchens, der gerade Fortsatz dagegen ist bedentend dicker und kann infolge dessen im Stämmchen weit verfolgt werden.

Die physiologische Bedeutung dieser von J. Arnold (8) zuerst entdeckten aber falsch gedeuteten Apparate ist erst von E h r li ch (1) richtig erkannt worden. Nach seiner Ansicht dienen sie dazu, um der Zelle Eindrücke zuzuführen, während der gerade Fortsatz sie von der Zelle zum Endorgan leitet. Oft beobachtet man, dass ein Nervenapparat von mehreren Fasern gebildet wird, d. h. dass eine Zelle in der Lage ist, von verschiedenen Quellen Eindrücke zu empfangen (Fig. 2). Andererseits findet man Fasern, die mehrere Seitenäste abgeben, welche an verschiedenen Zellen pericellulär endigen, d. h. eine Faser ist im Stande mehreren Zellen Impulse zu senden. Wir finden somit in der Harnblase dieselben Verbältnisse, die $S \mathrm{~m}$ i r n o w (3) in den anderen Nervenganglien des Frosches so klar und anschaulich dargestellt hat.

Die pericellulär endigenden Fasern lassen sich bäufig sehr weit verfolgen, wobei sie immer blass bleiben and innerhalb der Blasenwand nie eine Markscheide erhalten. Deshalb möchte ich annehmen, dass in dem von mir untersuchten Organe die pericellulären Apparate von marklosen, Remak'schen Fasern gebildet werden, obgleich ich nicht die Möglichkeit ausschliessen 
kann, dass sie ausserhalb der Blase markhaltig werden, cinmal weil es mir nicht gelungen ist eine solche Faser bis zur zugehörigen Zelle zu verfolgen, dann aber weil in anderen Organen die Zugehörigkeit der pericellulären Apparate zu markhaltigen Fasern direct nachgewiesen worden ist. Zuerst geschah es von Retzius, dann von Smirnow (3) beim Frosch und von A. Dogiel, Tim of ejew (5) und Plos ch ko (7) bei Süugern.

Was nun die anderen in der Harnblase vorkommenden Remak'schen Fasern anlangt, so gehören sie zu den motorischen und endigen in den glatten Muskelfasern. Diese Fasern verlaufen grösstentheils in Nervenstämmchen, weshalb ihr Ursprung nicht klargelegt werden kann. Doch findet man hin und wieder an gut gelungenen Präparaten solche Stellen, wo die Verhältnisse nicht so complieirt sind, dann bekommt man ein schönes Bild von einem automatischen Nervenmuskelapparat (Fig. 3).

Die Ansicht, dass die glatte Musculatur vom sympathisehen Nervensystem innervirt wird, wurde seit langer Zeit vertreten, doch beruhte sie ausschliesslich auf physiologischen Thatsachen. Der morphologische Nachweis des Zusammenhanges sympathischer Nervenzellen mit Muskelfasern iberhaupt wurde geliefert durch die Untersuchungen des Herrn Prof. C. Arnstein(11) und N. Lawd o w sky, die vor 15 Jahren angestellt wurden. Mittels der Chlorgoldmethode gelang es ihnen aus der Vorhofsscheidewand des Froschherzens solche Präparate herzustellen, an denen die Nervenfasern von ihren Zellen bis za den Muskelbündeln verfolgt werden konnten. Diese Beobachtungen sind alsdann von S mirnow (3) für die Herzmusculatur, von Plos chko (7) für die Trachealmusculatur und jetzt von mir für die Harnblasenmusculatur bestïtigt worden.

Ploschko (7) machte auch darauf aufmerksam, dass diese Fasern in ihrem Verlaufe nie markhaltig werden, sondern stets den Charakter der Remak'schen Fasern besitzen. Ich sah ebenfalls nie einen solchen Fortsatz myelinhaltig werden, so dass unsere Beobachtungen die Angaben von A. Dogiel, dass die Achseneylinder der motorischen sympathischen Zellen in markhaltige Fasern übergehen, nicht bestätigen.

Die in der Harnblase vorhandenen myelinhaltigen Fasern gehören zwei Categorien an: zur ersteren gehören die dünnen markhaltigen Fasern, die nach der gegenwärtig herrschenden Ansicht dem sympathischen Nervensystem zugezällt werden, zur 
zweiten die dicken markhaltigen Fasern des Cerebrospinalsystems. Erstere kommen bei weitem seltener in der Harnblase vor, als letztere; ihre Bedeutung, suwie auch ihr Ursprung bleibt noch immer räthselhaft. An einem Präparate sah ich aus einem Nervenstämmchen, welches aus marklosen und markhaltigen Fasern zusammengsetzt war, eine dünne myelinhaltige Faser austreten, eine Strecke weit isolirt verlanfen und dann sich in ein grosses breitmaschiges Netz von marklosen Fäden auflösen. Ob letzteres einen eigenartigen, vielleicht sensiblen, Nervenendapparat bildet, oder $o b$ es eine motorische Endigung ist, konnte nicht klargelegt werden.

Genauere Angaben können wir tuber die dickeren markhaltigen Fasern machen. Sie sind alle cerebrospinalen Ursprungs, sensibler Natul und besitzen einen eigenartigen Endapparat, der von E h r l i ch (1) zuerst als "Endbïumchen" beschrieben worden ist. Eh rl i c h berichtet, dass er in der Harnblase des Frosches sensible Nervenendapparate gesehen habe, $n$ die sich als ziemlich grosse rundliche Flecke präsentiren, die aus der successiven Theilung einer einzigen markhaltigen Nervenfaser hervorgehen. Die Terminalfasern dieser Verzweigungen tragen sämmtlich endständige Knöpfe". Diese Angaben sind dann u. A. von Cuccati und jüngst von Huber (Journal of comparative Neurology Vol. VII) bestätigt worden. Diese Endapparate sind in der Froschharnblase in grosser Menge vorhanden. Ich habe Präparate gesehen, wo das ganze Sehfeld von solchen Endbäumchen eingenommen war. Sie lagen entweder isolirt, wobei ihre charakteristische Form deutlich hervortrat, oder so dicht neben einander, dass man auf den ersten Blick glauben möchte, sie bilden ein Netziverk, doch überzeugt man sich mit Hülfe stärkerer Systeme, dass tuberall nur eine Ueberkreuzung, nicht aber Verschmelzung der Fäden stattfindet (Fig. 4).

Fig. 5 stellt einen isolirten Endapparat bei starker Vergrösserung dar. Die dicke myelinhaltige Nervenfaser tritt aus dem Stämmchen aus, begiebt sich nach oben, und indem sie allmählich immer dünner und dünner wird und ihre Markscheide verliert, zerfällt sie in eine Menge dünner variköser Fäden; die an ihren Enden knopfförmige Verdickungen tragen. Einen solchen Apparat möchten wir als ,terminales" Bäumchen bezeichnen, da mit dem Apparat auch die Faser aufhört, zum Unterschied von "lateralen" Apparaten, die von myelinlosen Collateralen mark- 
haltiger Fasern, welche an den Ranvier'schen Einschnürungen abgehen, gebildet werden.

Was nun die Lage der Endapparate anbetrifft, so bietet das Studium derselben in der Froschbarnblase einige Schwierigkeiten, da man keine Schnitte anfertigen kann. An Flächenpräparaten treten die in Rede stehenden Gebilde am schärfsten hervor, wenn man das Epithel entfernt, deshalb darf man annehmen, dass sie im subepithelialen Gewebe liegen. Intermuskuläre sensible Endapparate, wie sie Ag a b a b o w (4) im Ciliarkörper und Ploschko (7) in der Trachea beschrieben haben, und wie wir sie bei Säugern anch in der Harnblase gefunden haben, besitzt die Froschharnblase nicht. Dies lässt sich durch die Dünne der Wandung und das Fehlen verschiedener Muskelschichten leicht erklären.

\section{Die Innervation der Sängethierblase.}

Untersucht wurde die Harnblase von Maus, Ratte, Katze und Hund. Positive Resultate wurden nur bei dem letzterwähnten Thiere erhalten. Bei diesem kommt der N. vesicularis nach El l e nberger und $\mathrm{Baum}$ rom $\mathrm{N}$. haemorrhoidalis posterior. Auf seinem Wege zur Harnblase erhält er noch sympathische Fasern, welche rom Plexus hypogastricus stammen. Wie beim Frosch, so finden sich auch bei Säugern in der Blasenwandung Nervenganglien verschiedener Grösse, die sich recht schön mittels der E h r li c h'schen Methode demonstriren lassen. Zu diesem Ende macht man einem jungen Hund in der oben angegebenen Weise eine Injection, schneidet die Blase heraus und fillt sie mit einer physiologischen Kochsalzlösung. Dadurch wird die Blasenwand ausgedehnt, die Luft bekommt Zutritt zu allen Theilen derselben und die Färbung tritt scharf hervor. Man sieht alsdann schon mit unbewaffnetem Ange, noch besser aber mit der Lupe die intensir blau gefärbten Nervenganglien und die dazwischen liegenden grössseren Nervenstämmchen. Die Hauptmenge dieser Ganglien liegt an der Eintrittsstelle der Ureteren, dann am Hals und an den Seitentbeilen der Blase, dagegen sieht man keine am Vertex vesicae. Im Grossen und Ganzen begleiten sie die Blutgefässe. Ausser diesen verhältnissmässig grossen Ganglien finden sich noch zablreiche kleine, die nur unter dem Mikroskop sichtbar sind. Die Zellen dieser Ganglien sind gross, multipolar 
und lassen sich leichter als die des Frosches mit Methylenblau färben. Die Protoplasmafortsätze theilen sich und verlieren sich im Ganglion, während der Achsencylinderfortsatz für gewöhnlich in ein Stämmchen eintritt.

Was nun die Fasern der letzteren anlangt, so kann ich vorläufig nur $\mathfrak{a b e r}$ einen Theil der dicken markbaltigen Nervenfasern positive Angaben machen. Diese habe ich bis zu ihren Endapparaten verfolgt, was, soviel ich weiss, zum ersten Male gelungen ist. Die in Fig. 6 dargestellten Endapparate sind der Harnblase eines jungen Hundes entnommen. Sie sind nicht so complicirt, wie die des Frosches und erscheinen etwas mehr als jene in die Länge gezogen, was wohl mit der Faserrichtung des Stromas, in dem die Endbäumchen liegen, in Zusammenhang stehen dürfte.

Bis jetzt konnte ich nur intermusculäre Endapparate nachweisen, doch sind wahrscheinlich auch subepitheliale vorhanden.

Ueber die Nervenendigungen im Epithel selbst sei hier nur Folgendes bemerkt. R e t z i u s (10) beschreibt in der Harnblase des Kaninchens Nervenfasern, die in das Epithel eintreten und sich hier tangential verzweigen. Dabei treten sie ganz nahe an die Oberfläche heran, hören aber niemals hier auf, sondern kehren wieder in die Tiefe zurück, wo sie frei endigen. Auf diese Weise entstehen in den oberflächlichen Epithelschichten Nervenbögen, die nach $R$ etzi us deshalb vorhanden sind, weil die Blase verschieden gedehnt wird.

Ich beobachtete im Epithel der Katzenblase noch andere Endapparate, welche die Zellen umgeben (Fig. 7). Ihrem morphologischen Charakter nach ähneln sie denjenigen, welche $\mathrm{T}$ i m ofejew (6) an den Epithelzellen des Vas deferens und an den unteren Zellen des Stratum Malpighii der Haut der Glans penis beim Kaninchen und Plos ch k o (7) in der untern Schicht des vielschichtigen Epithels der Epiglottis beschrieben haben. Prof. A r $\mathbf{n}$ s t e i n ist geneigt in diesen Apparaten pericelluläre Nervenendapparate $\mathrm{zu}$ sehen, die denjenigen analog sind, welche er an den Drüsenzellen beschrieben hat.

Zum Schluss möchte ich noch erwähnen, dass die Frage über den Nervenapparat der Harnblase in jüngster Zeit besonderes Interesse gewonnen hat, dank den Untersuchungen, welche auf diesem Gebiete von physiologischer Seite vorgenommen worden 
sind. So studierte $\mathrm{Z}$ e is s l (13) das Verhalten der entnervten Blase. Er durchschnitt bei Hunden die beiden NN. hypogastrici und errigentes. Bei den Thieren, welche die Operation überlebten, funktionirte die Blase vollkommen normal. Daraus schloss er, dass in der Blasenwand Nervenapparate da sein müssen, welche automatisch wirken können, oder dass die Blase noch von anderer Seite innerviert wird. Letzteres wird jedoch durch folgenden Umstand widerlegt: wenn man gleichzeitig beide NN. splanchnici reizt, erfolgt eine Zusammenziehung des Detrusors und Eröffnung. des Sphincters. Wenn man aber die erwähnten Nerven nach der Durchschneidung der beiden NN. liypogastrici und errigentes reizt, so bleibt der Effect aus. Es folgt daraus, dass die Reize, un vom Splanchnicus zur. Blase zu gelangen, die beiden letztgenannten Nerven passiren müssen.

Goltz und Ewald (14) untersuchten, in wiefern die operative Entfernung verschiedener Rïckenmarksabschnitte Functionsstörungen in den verschiedenen Organen hervorruft. Durch eine grosse Anzahl von Versuchen haben sic zunächst feststellen können, dass ein Hund am Leben bleibt and Jahre lang vollständig gesund sein kann, wenn er von den grossen Nervencentren nur noch das Gehirn und Halsmark beibehält. Die Blase wird nur unmittelbar nach der Entfernung des Lenden- und des Kreuzbeinmarks funktionsunfälig: sie wird durch angesammelten Harn so weit ausgedehnt, dass es nothwendig wird, von Zeit zu Zeit ihren Inhalt durch vorsichtigen Druck auf die Bauchdecken zn entleeren. Allmählich aber bessert sich der Lähmungszustand, man bemerkt, dass das Thier längere Zeit trocken bleibt und der Harn von selbst in grösseren Mengen ausgetrieben wird. Nach Verlauf von noch einiger Zeit funktionirt die Blase ganz normal.

Auf Grund dieser physiologischen Thatsachen mïssten wir annehmen, dass in der Blase ein selbstständiger vom Cerebrospinalsystem unabhängiger Reflexbogen vorhanden ist, so wie es A. Dog i e l (12) in einigen anderen Organen (im Ganglion stellatum, in allen Ganglien des Brusttheiles des Sympathicus, im Ganglion coeliacum, in den Ganglien der Auerbach'schen und der Meissner'schen Geflechte) nachgewiesen haben will. Alle diese Nerrenganglien sollen nämlich nach seiner Ansicht zweierlei Arien von sympathischen Zellen enthalten: sensible und mo- 
torische, die mit einander folgendermaassen verbunden sind: der Achsencylinderfortsatz einer motorischen Zelle endet in den glatten Muskelfasern, während die Protoplasmafortsätze sich wiederholt theilen und im Ganglion verästeln. Mit diesen Verästelungen tritt in Contact der Achsencylinderfortsatz der sensiblen Zellen entweder direct durch seine Endverzweigungen oder durch die seiner Collateralen. Auf diese Weise entsteht im Ganglion ans den protoplasmatischen Fortsätzen der motorischen Zellen und den Endzweigen der Achsencylinder der sensiblen Zellen ein Nervengeflecht, das A. D o g i e l (12) intercelluläres nennt. Was nun die Protoplasmafortsätze der letzterwähnten Zellen anlangt, so konnte er sie bis zur Mucosa und Submucosa verfolgen. Thre Endapparate sah er nicht, doch ist er der Meinung, dass sie ebensolche Endapparate besitzen, wie die myelinhaltigen cerebrospinalen Nervenfasern.

Wie wir nun gesehen haben, lässt sich in der Harnblase ein ähnlicher automatischer Reflexbogen anatomisch nicht nachweisen. Anch scheint uns A. Dogiel für die von ihm untersuchten Organe diesen Beweis nicht geliefert zu baben, da es ihm nicht gelungen ist, die Achsencylinderfortsïtze seiner sensiblen Zellen dircet von den Zellkïrpern bis zu den EndverïsteIungen zu verfolgen, welche in Contact treten sollen mit den Enden der protoplasmatischen Fortsätze seiner motorischen Zellen.

Zum Schluss möchte ich noch dem Wirklichen Staatsrath Herrn Professor Dr. C. A r n s te in für die Anregung zu dieser Arbeit und die liebenswürdige Unterstützung während der Ausführung derselben auch an dieser Stelle meinen herzlichsten Dank aussprechen.

\section{Literatur-Verzeichniss.}

1. Ehrlich, P., Ueber die Methylenblau-Reaction auf die lebende Nervensubstanz. (Deutsche medicinische Wochenschrift, 1886, Nr. 4, Vortrag vom 21. December 1885.)

2. C. Arnstein, Die Methylenblatt-Färbung als histologische Methode (Anatomischer Anzeiger Nr. 5 u. Nr. 17, 1887.)

3. Sinirnow, A., Beiträge zur Histologie des peripheren Nervensystems der Batrachier. (Inaug.-Diss. Kasan, 1891. Russisch.) 
4. Ag a babow, A., Ueber dic Nervenendigungen des Ciliarkörpers bei den Säugethieren und beim Menschen. (Inaug.-Diss. 1893, Kasan. Russisch.)

5. Timofejew, D., Ueber die Nervenendigungen der männlichen Geschlechtsorgane bei den Säugethieren und Menschen. (Inaug.Diss. 1896, Kasan. Russisch.)

6. Derselbe, Ueber eine besondere Art von eingekapselten Nervenendigungen in den männlichen Geschlechtsorganen b. Säugethieren. (Anat. Anz. Bd. XI, Nr. 2, 1895.)

7. Ploschko, A., Ueber die Nervenendigungen im Larynx und in der Trachea der Säugethiere. (Inaug.-Diss. Kasan, 1896. Russisch. Anat. Anz. Bd. XIII. Nr. 1 u. 2 1897.)

8. J. Arnold, Zar Histologie der Lunge. (Virchow's Arch. Bd. 28. 1863. H. 5 u. 6.)

9. Derselbe, Ueber die feineren Verhältnisse der Ganglienzellen in dem Sympathicus des Frosches. (Vir ch. Arch. Bd. 32. H. 1. 1865.)

10. Retzius, G., Biologische Untersuchungen. Neue Folge IV, 1892.

11. C. Arnstein und N. Lawdowsky, Ueber die Fortsätze der Nervenzellen in den Herzganglien. (Arch. f. mikr. Anat. Bd. 29.)

12. A. Dogiel, Zur Frage über den feineren Bau des sympathischen Nervensystems bei den Säugethieren. (Arch. f. mikr. Anat. Bd. 46, 1886). Zwei Arten sympathischer Zellen. (Anat. Anzeiger Bd. II, Nr. 22. 1895.)

13. Z e issl, M. v., Ueber die entnervte Blase. (Wiener klin. Wochenschrift Nr. 20. 1896.)

14. Goltz u. Ewald, Der Hund mit dem verkürzten Rückenmarke. ( $P$ fl ü ger's Archiv Bd. 63. 1896.)

\section{Erklärung der Abbildungen auf Tafel I.}

Fig. 1. Ganglienzelle aus der Wand der Froschharnblase mit geradem Fortsatz und pericellulärem Apparat. Methylenblauinjection, $\mathrm{Z}$ e is s, Homog. Immers. $1 / 12$ Comp. Ocul. 6.

Fig. 2. Zwei Ganglienzellen aus der Wand der Froschbarnblase. Die pericellulären Apparate werden von verschiedenen Nervenfasern gebildet. Methylenblauinjection, $\mathrm{Z}$ e is s, Homog. Imm. $1 / 12$ Comp. Ocul. 6.

Fig. 3. Drei Ganglienzellen aus der Wand der Froschbarnblase. Der gerade Fortsatz endigt in der Muskulatur, die pericellulären Apparate werden von der Nervenfaser, welche aus dem Stämmchen heraustritt, gebildet. Methylenblauinjection, Z e is s, Oc. IV, Obj. Trockenapochr. 3,0.

Fig. 4. Subepitheliale Endbäumchen aus der Wand der Froschharnblase im Zusammenhang mit den sie bildenden Nervenfasern. Methylenblauinjection, Z e is s, Ocul. IV, Obj. A.A. 
Fig. 5. Subepitheliales Endbäumchen aus der Wand der Harnblase des Frosches bei starker Vergrösserung ( $\mathrm{Z}$ e i s s, Comp. Oc. 6 . Obj. 8,0).

Fig. 6. Intramuskuläre Endapparate aus der Wand der Harnblase des Hundes. Methylenblauinject. Z e is s, Ocul. IV, Obj. D.

Fig. 7. Katze. Blasenepithelzellen mit pericellulären Apparaten. Methylenblauinjection. Zeiss, Homog. Imm. Oc. 8. Tubusl. 16.

(Aus der histologischen Anstalt des Carolinischen Institutes

in Stockholm.)

\section{Studien über Neuroglia.}

Von

Erik Muller.

Hierzu Tafel II-V und 1 Textfigur.

Das in physiologischer und pathologischer Hinsicht gleich wichtige Gewebe, welches von Rudolf Virchow 1846 im centralen Nervensystem entdeckt und von ihm später Neuroglia genannt wurde, hat eine umfassende Geschichte aufzuweisen, ohne dass wir deshalb zu einer annähernd abgeschlossenen Kenntniss desselben gelangt sind. Dieses hat ohne $\mathrm{Z}_{w}$ eifel seinen Grund in den Schwierigkeiten, die die Darstellung der Neuroglia in technischer Hinsicht darbietet, Schwierigkeiten, welche sie mit ihrer Schwester, dem eigentlichen Nervengewebe, in hohem Grade theilt. Die Geschichte der Neuroglia lässt sich darum in verschiedene Perioden eintheilen, die in der nächsten Beziehung zu der Entwicklung der histologischen Technik stehen. Unsere gegenwärtige Kenntniss der Neuroglia verdanken wir vor allem der Anwendung zweier ganz versehiedener Methoden, die man nach ihren Erfindern als die Golgi'sche und die Weigert'sche bezeichnet. Der grosse Fortschritt, der diese beiden Lehren von der Neuroglia, die diesen Methoden ihr Entstehen verdanken, gegenüber den früheren Lehren charakterisirt, besteht vor allem 